\title{
Radioguided Occult Lesion Localization Versus Wire- guided Localization of Nonpalpable Breast Lesions: A Comparative Analysis
}

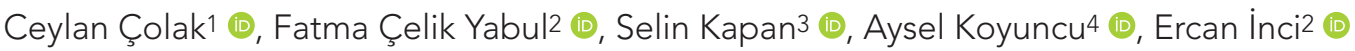 \\ ${ }^{1}$ Imaging Institute, Clinic of Cleveland, Cleveland, ABD \\ 2University of Health Sciences Turkey, İstanbul Bakırköy Dr. Sadi Konuk Training and Research Hospital, Clinic of Radiology, İstanbul, Turkey \\ ${ }^{3}$ University of Health Sciences Turkey, İstanbul Bakırköy Dr. Sadi Konuk Training and Research Hospital, Clinic of General Surgery, İstanbul, Turkey \\ ${ }^{4}$ University of Health Sciences Turkey, İstanbul Bakırköy Dr. Sadi Konuk Training and Research Hospital, Clinic of Nuclear Medicine, İstanbul, Turkey
}

Cite this article as: Çolak C, Yabul F, Kapan S, Koyuncu A, İnci E. Radioguided Occult Lesion Localization Versus Wire-guided Localization of Nonpalpable Breast Lesions: A Comparative Analysis. JAREM 2020;10(1): 88-93

\begin{abstract}
Objective: Wire-guided localization (WGL) is the preoperative localization method most commonly used before the surgical excision of non-palpable breast lesions (NPBLs). Recently, radioguided occult lesion localization (ROLL) has emerged as an alternative to WGL. We sought to compare the efficacy of ROLL with that of WGL for the preoperative localization of NPBLs and to assess our experience encountered as ROLL is implemented at our institution.

Methods: We retrospectively identified reports of patients with NPBLs who underwent mammography- or ultrasonography-guided ROLL or WGL between January 2014 and March 2017. Medical records were reviewed to compare radiologic and pathologic findings, rates of accurate localization, specimen volumes, lengths of operation, creation of positive surgical margins, number of simultaneous sentinel lymph node biopsies (SLNB) performed, complication rates, and lengths of hospital stay.

Results: Our search identified 67 women (mean age, 52.7 years; range, 32-69 years) diagnosed with NPBLs during the study period. ROLL was used in 25 patients; WGL was used in 42 patients. Both methods had a high accurate localization rate (ROLL, 96\%; WGL, 98\%). The length of operation was longer in the ROLL group than in the WGL group $(p=0.001)$, and more SLNBs were performed in the ROLL group than in the WGL group. No significant differences were seen between the groups in terms of radiologic and pathologic findings, specimen volumes, positive surgical margins, complication rates, or lengths of hospital stay.
\end{abstract}

Conclusion: ROLL is a promising alternative to WGL for preoperative localization of NPBLs. The operation time for ROLL procedures at our institution will likely decrease as clinicians become more familiar with the procedure.

Keywords: Nonpalpable breast lesion, ROLL, WGL

\section{INTRODUCTION}

Breast lesions that cannot be palpated on physical examination but are found to have features suggestive of malignancy on imaging studies are known as nonpalpable breast lesions (NPBLs) (1). Over the past 20 years, the detection of NPBLs has increased (1-6); this is important, as early detection of NPBLs can substantially reduce both morbidity and mortality (7-9).
NPBLs must be accurately localized before surgical excision is attempted (1). The main aim of localization is to allow for total excision of the lesion with minimal tissue loss. Wire-guided localization (WGL) is the most commonly used technique for lesion localization (10). However, this method can be complicated by the breakdown of the wire, difficulties with insertion, and wire dislodgement and migration, which can lead to pain and pneumothorax (11-13).

ORCID IDs of the authors: C.Ç. 0000-0002-8278-9302; F.Ç.Y. 0000-0002-0156-90056; S.K. 0000-0001-9339-4894; A.K. 0000-0001-8370-3526; E.I. 0000-0002-3791-2471. 
localization needle, and a control chart was used to determine whether the wire was in the lesion. Specimen graphy was also obtained to determine whether the lesion could be surgically excised.

In ultrasonography-guided WGL, an Aplio 500 system (Toshiba Medical Systems Corp., Tokyo, Japan) was used for breast marking. With the patient in the supine position, a local anesthetic was applied to the area near the lesion. Using ultrasonographic guidance, the radiologist advanced the marking needle and then the wire into the lesion, and surgical excision was performed.

The technique used for mammography-guided ROLL was similar to that used for mammography-guided WGL. With ROLL, however, marking was performed via the intrathecal injection of radioactive material through a $20 \mathrm{G}$ needle. The radiologist injected $0.5 \mathrm{mCi}$ Tc$99 \mathrm{~m}$-macroaggregate albumin (MAA) in a volume of 0.2 to $0.3 \mathrm{~mL}$. Subsequently, $0.2 \mathrm{~mL}$ of water-soluble nonionic contrast material was injected to determine whether the lesion had been accurately localized, and mammography was performed to determine whether the area of suspicion could be surgically excised.

The technique used for ultrasonography-guided ROLL was also similar to that used for ultrasonography-guided WGL. The only difference was that lesions were localized with ultrasonography while the radionuclide was injected to ensure that increased echogenicity in the lesion was seen. For surgical excision with ROLL, general anesthesia was administered to patients in the operating room, and radioactivity was measured as a hot spot with an intraoperative gamma probe (Crystal Probe System SG04; Crystal Photonics, Berlin, Germany). The highest hot spot was then selected for excision on skin that was marked with a marking pen, and this region was excised. Radioactivity control of the excised area was assessed with a gamma probe to ensure that no radioactive tissue remained.

The ROLL and WGL groups were compared in terms of radiologic and pathologic findings, rates of accurate localization, specimen volumes, lengths of operation, creation of positive surgical margins, number of SLNBs performed, complication rates, and lengths of hospital stay.

\section{Evaluation of Lesion Localization Success}

For patients undergoing mammography-guided ROLL, successful lesion localization was defined as observation of the radioactive contrast material near the lesion in question. For patients undergoing ultrasonography-guided ROLL, successful localization was defined as an increase in echogenicity in the lesion when radioactive material was administered (Figure 1). Before the surgery, scintigraphic control could be performed to ensure that the radionuclide had not spread (Figure 2); in this study, only one patient had such a scintigraphic image available. In terms of the specimen, lesions localized with mammography were examined by specimen graphy to determine whether the marked lesion was removed during surgery (Figure 3). Lesions localized with ultrasonography were assessed with a gamma probe while the radionuclide was injected to ensure that increased echogenicity positions. A wire in the form of a hook was fixed inside the 
in the lesion was seen (Figure 4).

For patients undergoing mammography-guided WGL, successful lesion localization was defined as the presence of the wire tip near the lesion in question $(<1 \mathrm{~cm})$. For those undergoing ultrasonography-guided WGL, successful lesion localization was defined as the presence of echogenicity of the wire in the lesion. For patients who underwent mammography-guided WGL, observation of the suspicious lesion on specimen control graphy was considered indicative of successful lesion removal (Figure 5). No radiologic examinations of the specimens were performed for patients who underwent ultrasonography-guided WGL.

\section{Statistical Analysis}

Mean, standard deviation, and median values for continuous variables and frequency and percentage values for categorical variables were calculated. A chi-square test, Fisher's exact test, and the Fisher-Freeman-Halton exact test were used to assess

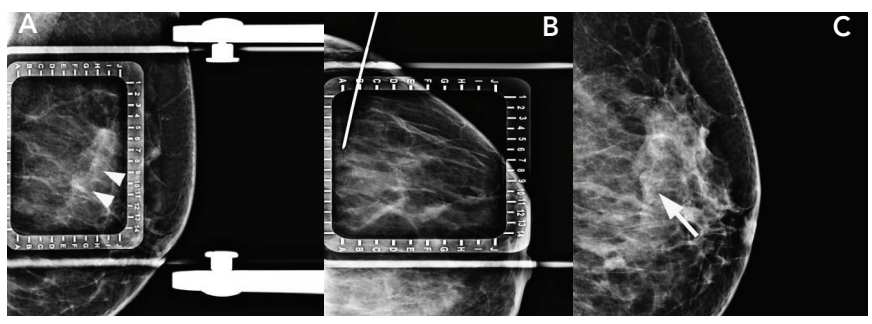

Figure 1. Radioguided occult lesion localization performed with mammographic guidance. A. Microcalcifications (arrowheads) can be seen. B. Needle can be seen entering the suspected area. C. After injection of contrast, increased density can be seen (arrow)
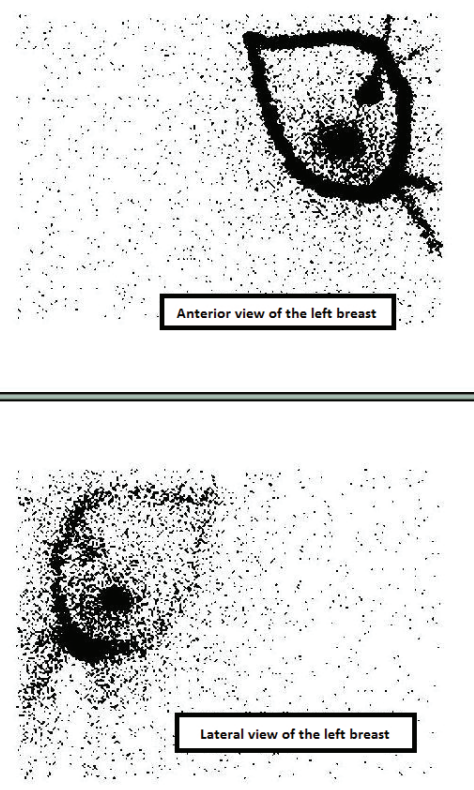

Figure 2. Scintigraphic graphy of lesion obtained using radioguided occult lesion localization under ultrasonographic guidance before surgical excision categorical interrelationships. Normal distribution was assessed with the Kolmogorov-Smirnov test. An independent-samples t-test was used when normal distribution was observed for continuous variables, whereas a Mann-Whitney U-test was used when normal distribution was not observed. For all statistical tests, $p<0.05$ was considered statistically significant.

\section{RESULTS}

Our search identified 67 women (mean age, 52.7 years; range, 32-69 years) who had been diagnosed with NPBL with features suggestive of malignancy during the study period. ROLL was used
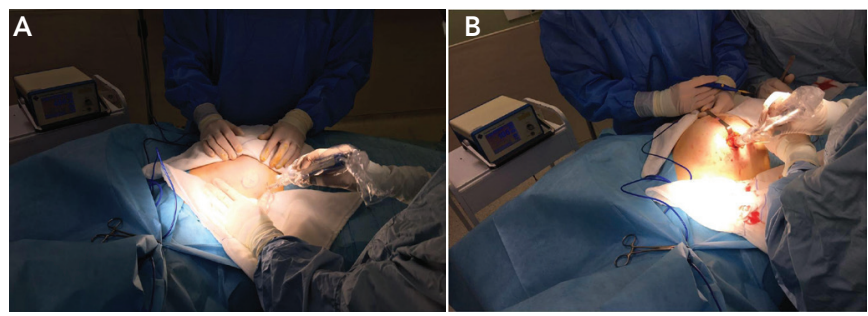

Figure 3. Use of a gamma probe (A) before surgical excision to localize the lesion and (B) after lesion removal to assess the cavity for evidence of residual lesion tissue
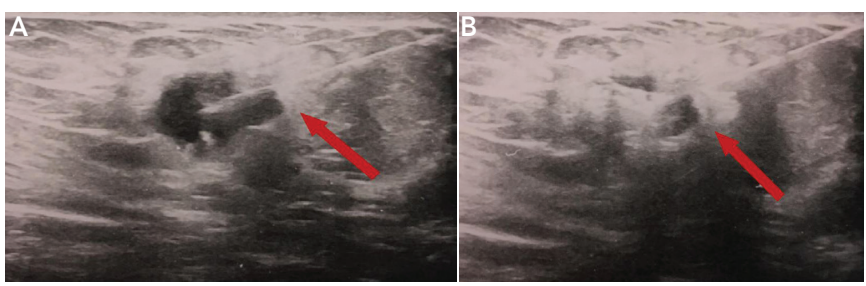

Figure 4. Radioguided occult lesion localization performed with ultrasonographic guidance. A. The needle can be seen inside the lesion (red arrow). B. After radionuclide injection, there is increased echogenicity in the lesion (red arrow)

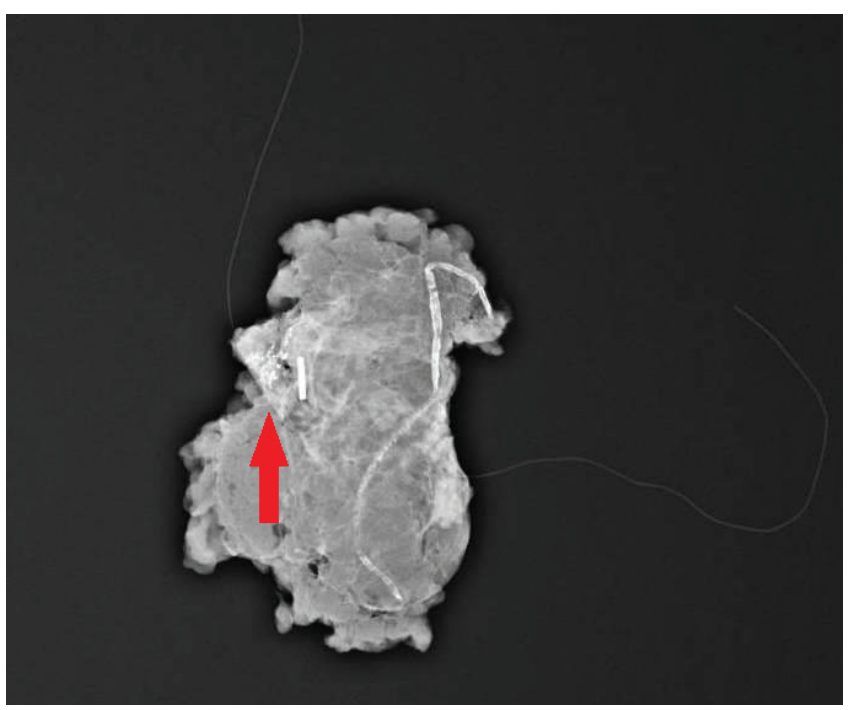

Figure 5. The control graphy for a specimen obtained using wireguided localization shows the wire in the area of the calcification (red arrow) 
The WGL method of lesion localization has been used successfully for many years. In this study, we assessed whether the ROLL technique, just recently introduced at our institution, could provide safe and effective lesion localization, and we found that ROLL accurately localized NPBLs in $96 \%$ of patients, a rate similar to that seen with WGL (98\%). These rates are also comparable to the localization rates of $89 \%$ to $100 \%$ reported in previous studies (17-22).

Accurate lesion localization and early surgical excision of NPBLs are important, as NPBLs are malignant in 10\% to $30 \%$ of patients. BI-RADS 4 and 5 lesions carry the highest risk of malignancy $(16,23,24)$. In this study of patients with BI-RADS 4 or 5 lesions, BI-RADS 4 lesions demonstrated heterogeneous distribution of malignancy risk in both the ROLL and WGL groups. The American College of Radiology states that the BI-RADS subgroups of $4 a, 4 b$, and $4 c$ have malignancy risks of $6 \%, 15 \%$, and $53 \%$, respectively (25), but the radiologic reports for lesions in this study did not include BI-RADS 4 subclassifications. For patients with BI-RADS 5 lesions, the malignancy rates in both the ROLL and WGL groups were consistent with rates previously reported (25).

We observed no difference between the groups in the creation of positive surgical margins and in the size of specimens obtained. In previous studies, the ROLL procedure was associated with less involved margins and smaller surgical specimens than the WGL technique (20,25-29). Because the surgeons at our institution have just begun using ROLL, a larger excision than usual may have been made, leading to larger specimen sizes. The size of the margins created is also dependent on lesion size and histologic grade; more involved margins are seen more frequently with cases of large ductal carcinoma in situ and lobular carcinoma in situ $(28,30)$. The suggested protocol for creating surgical margins in patients with NPBLs is to use a gamma probe to trace the radioactivity in the center of the lesion and then assess the excision site to ensure that no residual lesion tissue remains $(15,17,18,31,32)$, as we did in this study. However, some authors recommend excising an additional 1 to $2 \mathrm{~cm}$ of tissue around the maximal radioactive site $(20,33)$.

The length of the operation was longer with ROLL than with WGL in our study. These results again contrast with those of previous studies, which reported shorter surgery times with ROLL than with WGL $(17,18,27,32)$. This difference may again be partly explained by the learning curve required when implementing the ROLL technique. Additionally, more patients in the ROLL group than in the WGL group underwent SLNB, which may have added to the procedure time.

Complication rates and lengths of hospital stay were similar for the 2 groups and were similar to results from previous studies (17). Complications such as breakdown of the markers, syncope, pain, and pneumothorax have previously been reported with WGL (1012), but a meta-analysis found that no major complications have been reported with either WGL or ROLL (27).

In this study, most of the ROLL procedures were guided by ultrasonography; this choice was determined by the radiologist. 
In general, the choice of imaging guidance depends on the availability of the technique and on the lesion characteristics. Mammography is recommended for assessing microcalcifications, whereas ultrasonography is recommended for evaluating solid and cystic lesions (18). Additionally, the choice of imaging used for guidance should be based on the type of imaging that first demonstrated the lesion (34).

In both the WGL and ROLL groups, SLNB was performed in only certain patients. However, more patients in the ROLL group underwent SLNB, perhaps because benign lesions were more common in the WGL group and lesions in the ROLL group were more likely to be mass-like. Histopathologic analysis of SLNB results was not included in this study.

Our study had several limitations. The main limitation of this study was that it was a retrospective study with small sample size, which limited our ability to include more lesions. Prospective data are needed to confirm these findings. On the other hand, study patients were treated by various radiologists and surgeons who had different degrees of experience with the procedures involved. These variations may have affected our analysis regarding accurate localization of lesions. Nuclear medicine at our institution has been a promising deparment which has provided us radionuclide material for ROLL. Collaboration of the departments of radiology and nuclear medicine has given us more chance to implement this new technique in our institution. This study was our first experience of this collaboration with some difficulties including low number of scintigraphic controls after ROLL procedures. Another limitation was that we could not evaluate the patient's comfort because of the retrospective design of the study.

\section{CONCLUSION}

In conclusion, this study demonstrated that the ROLL technique is a safe and effective method for preoperatively localizing NPBLs, thus allowing accurate surgical excision of potentially malignant lesions. The ROLL technique may therefore serve as an alternative to WGL for the localization of NPBLs, as this technique is simple to perform (even for inexperienced operators) and provides satisfactory results.

Acknowledgment: This study was performed in University of Health Sciences Turkey, İstanbul Bakırköy Dr. Sadi Konuk Training and Research Hospital over the study period. We thank our scientific medical writer, Megan Griffiths, ELS, for her help with editing this paper. Informed consent was obtained for the Figure $3 \mathrm{~A}$ and $3 \mathrm{~B}$.

Ethics Committee Approval: Ethics committee approval was received for this study from the ethics committee of University of Health Sciences Turkey İstanbul Bakırköy Dr. Sadi Konuk Training and Research Hospital (approval number: 2017/29).

Informed Consent: Informed consent was not taken from patients due to the retrospective nature of the study.

Peer-review: Externally peer-reviewed.

Author Contributions: Surgical and Medical Practices - C.Ç., S.K., A.K.; Concept - C.Ç., F.Ç.Y., S.K., A.K., E.I.; Design - C.Ç., S.K., A.K., E.I.; Data Collection or Processing - C.Ç., F.Ç.Y., S.K., A.K.; Analysis or Interpretation - C.Ç., F.Ç.Y., S.K., A.K., E.I.; Literature Search - C.Ç., F.Ç.Y.; Writing - C.Ç., S.K., A.K., E.I.
Conflict of Interest: The authors have no conflict of interest to declare.

Financial Disclosure: The authors declared that this study has received no financial support.

\section{REFERENCES}

1. Markopoulos C, Kakisis J, Kouskos S, Kontzoglou K, Koufopoulos K, Gogas J. Management of nonpalpable, mammographically detectable breast lesions. World J Surg 1999; 23: 434-8.

2. Altomare V, Guerriero G, Giacomelli L, Battista C, Carino R, Montesano $M$, et al. Management of nonpalpable breast lesions in a modern functional breast unit. Breast Cancer Res Treat 2005; 93: 85-9.

3. Cady B, Stone MD, Schuler JG, Thakur R, Wanner MA, Lavin PT. The new era in breast cancer. Invasion, size, and nodal involvement dramatically decreasing as a result of mammographic screening. Arch Surg 1996; 131: 301-8.

4. Wilson M, Boggis CR, Mansel RE, Harland RN. Non-invasive ultrasound localization of impalpable breast lesions. Clin Radiol 1993; 47: 337-8.

5. Ahmed M, Rubio IT, Klaase JM, Douek M. Surgical treatment of nonpalpable primary invasive and in situ breast cancer. Nat Rev Clin Oncol 2015; 12: 645-63.

6. Marinovich ML, Azizi L, Macaskill P, Irwig L, Morrow M, Solin LJ, et al. The association of surgical margins and local recurrence in women with ductal carcinoma in situ treated with breast-conserving therapy: a metaanalysis. Ann Surg Oncol 2016; 23: 3811-21.

7. $\mathrm{Li} \mathrm{Cl}$, Daling JR, Malone KE. Age-specific incidence rates of in situ breast carcinomas by histologic type, 1980 to 2001. Cancer Epidemiol Biomarkers Prev 2005; 14: 1008-11.

8. Perdue P, Page D, Nellestein M, Salem C, Galbo C, Ghosh B. Early detection of breast carcinoma: a comparison of palpable and nonpalpable lesions. Surgery 1992; 111: 656-9.

9. Harvey JA, Nicholson BT, Cohen MA. Finding early invasive breast cancers: a practical approach. Radiology 2008; 248: 61-76.

10. Frank HA, Hall FM, Steer ML. Preoperative localization of nonpalpable breast lesions demonstrated by mammography. N Engl J Med 1976; 295: 259-60.

11. Homer MJ, Smith TJ, Safaii H. Prebiopsy needle localization. Methods, problems, and expected results. Radiol Clin North Am 1992; 30: 139-53.

12. Rappaport W, Thompson S, Wong R, Leong S, Villar H. Complications associated with needle localization biopsy of the breast. Surg Gynecol Obstet 1991; 172: 303-6.

13. Mariscal Martinez $A$, Solà $M$, de Tudela AP, Julián JF, Fraile M, Vizcaya $\mathrm{S}$, et al. Radioguided localization of nonpalpable breast cancer lesions: randomized comparison with wire localization in patients undergoing conservative surgery and sentinel node biopsy. AJR Am J Roentgenol 2009; 193: 1001-9.

14. Bernardi S, Bertozzi S, Londero AP, Gentile G, Giacomuzzi F, Carbone A. Incidence and risk factors of the intraoperative localization failure of nonpalpable breast lesions by radio-guided occult lesion localization: a retrospective analysis of 579 cases. World J Surg 2012; 36: 1915-21.

15. Luini A, Zurrida S, Paganelli G, Galimberti V, Sacchini V, Monti S, et al. Comparison of radioguided excision with wire localization of occult breast lesions. Br J Surg 1999; 86: 522-25.

16. van Rijk MC, Tanis PJ, Nieweg OE, Loo CE, Olmos RV, Oldenburg HAS, et al. Sentinel node biopsy and concomitant probe-guided tumor excision of nonpalpable breast cancer. Ann Surg Oncol 2007; 14: 627-32.

17. Medina-Franco H, Abarca-Pérez L, Garcia-Alvarez MN, Ulloa-Gómez JL, Romero-Trejo C, Sepúlveda-Méndez J. Radioguided occult lesion localization (ROLL) versus wire-guided lumpectomy for non-palpable breast lesions: a randomized prospective evaluation. J Surg Oncol 2008; 97: 108-11.

18. Moreno M, Wiltgen JE, Bodanese B, Schmitt RL, Gutfilen B, da Fonseca LMB. Radioguided breast surgery for occult lesion localization-correlation between two methods. J Exp Clin Cancer Res 2008; 27: 29.

19. Ocal K, Dag A, Turkmenoglu O, Gunay EC, Yucel E, Duce MN. Radioguided occult lesion localization versus wire-guided localization for non-palpable breast lesions: randomized controlled trial. Clinics (Sao Paulo) 2011; 66: 1003-7.

20. Duarte C, Bastidas F, de los Reyes A, Martínez MC, Hurtado G, Gómez $\mathrm{MC}$, et al. Randomized controlled clinical trial comparing radioguided occult lesion localization with wire-guided lesion localization to evaluate 
their efficacy and accuracy in the localization of nonpalpable breast lesions. Surgery 2016; 159: 1140-5.

21. Alamdaran SA, Farokh D, Haddad AS, Daghighi N, Modoodi E, Sadeghi R, et al. Assessment of Ultrasound/Radio-guided Occult Lesion Localization in Non-palpable Breast Lesions. Asia Ocean J Nucl Med Biol 2018; 6: 10-4.

22. Pisano ED, Fajardo LL, Tsimikas J, Sneige N, Frable WJ, Gatsonis CA, et al. Rate of insufficient samples for fine-needle aspiration for nonpalpable breast lesions in a multicenter clinical trial: The Radiologic Diagnostic Oncology Group 5 Study. The RDOG5 investigators. Cancer 1998; 82: 679-88.

23. Schwartz GF, Feig SA, Patchefsky AS. Significance and staging of nonpalpable carcinomas of the breast. Surg Gynecol Obstet 1988; 166: 6-10.

24. Skinner MA, Swain M, Simmons R, McCarty KS Jr, Sullivan DC, Iglehart JD. Nonpalpable breast lesions at biopsy. A detailed analysis of radiographic features. Ann Surg 1988; 208: 203-8.

25. Lazarus E, Mainiero MB, Schepps B, Koelliker SL, Livingston LS. BI-RADS lexicon for US and mammography: interobserver variability and positive predictive value. Radiology 2006; 239: 385-91.

26. Feggi L, Basaglia E, Careione S, Querzoli P, Soliani G, Ascanelli S, et al. An original approach in the diagnosis of early breast cancer: use of the same radiopharmaceutical for both non-palpable lesions and sentinel node localisation. Eur J Nucl Med 2001; 28: 1589-96.

27. Sajid MS, Parampalli U, Haider Z, Bonomi R. Comparison of radioguided occult lesion localization (ROLL) and wire localization for non-palpable breast cancers: a meta-analysis. J Surg Oncol 2012; 105: 852-58.
28. Tartter PI, Bleiweiss IJ, Levchenko S. Factors associated with clear biopsy margins and clear reexcision margins in breast cancer specimens from candidates for breast conservation. J Am Coll Surg 1997; 185: 268-73.

29. Nadeem R, Chagla LS, Harris O, Desmond S, Thind R, Titterrell C, et al. Occult breast lesions: a comparison between radioguided occult lesion localisation (ROLL) vs. wire-guided lumpectomy (WGL). Breast 2005; 14: 283-89.

30. Van Esser S, Hobbelink M, Van der Ploeg IM, Mali WP, Van Diest PJ, Borel Rinkes $\mathrm{IH}$, et al. Radio guided occult lesion localization (ROLL) for nonpalpable invasive breast cancer. J Surg Oncol 2008; 98: 526-29.

31. Machado RH, Oliveria AC, Rocha AC, Landesmann MC, Martins FP, Lopes $\mathrm{SA}$, et al. Radioguided occult lesion localization (ROLL) and excision of breast lesions using technetium-99m-macroaggregate albumin and air injection control. J Exp Clin Cancer Res 2007; 26: 323-7.

32. Rampaul RS, Bagnall M, Burrell H, Pinder SE, Evans AJ, Macmillan $\mathrm{RD}$. Randomized clinical trial comparing radioisotope occult lesion localization and wire-guided excision for biopsy of occult breast lesions. Br J Surg 2004; 91: 1575-7.

33. Sarlos D, Frey LD, Haueisen H, Landmann G, Kots LA, Schaer G. Radioguided occult lesion localization (ROLL) for treatment and diagnosis of malignant and premalignant breast lesions combined with sentinel node biopsy: a prospective clinical trial with 100 patients. Eur J Surg Oncol 2009; 35: 403-8.

34. Zurrida S, Galimberti V, Monti S, Luini A. Radioguided localization of occult breast lesions. Breast 1998; 7: 11-3. 